\title{
Dysmobility syndrome: current perspectives
}

This article was published in the following Dove Press journal:

Clinical Interventions in Aging

16 January 2017

Number of times this article has been viewed

\section{Keith D Hill' \\ Kaela Farrier' \\ Melissa Russell ${ }^{2}$ \\ Elissa Burton'}

'School of Physiotherapy and Exercise Science, Faculty of Health Sciences, Curtin University, Perth, WA, Australia; ${ }^{2}$ Department of Epidemiology and Biostatistics, School of Population and Global Health, The University of Melbourne, Parkville, VIC, Australia
Correspondence: Keith D Hill School of Physiotherapy and Exercise Science, Faculty of Health Sciences, Curtin University, GPO Box U1 987, Perth, WA 6845, Australia

Tel +6I 892663618

Fax +6I 892662399

Email keith.hill@curtin.edu.au
Background: A new term, dysmobility syndrome, has recently been described as a new approach to identify older people at risk of poor health outcomes. The aim was to undertake a systematic review of the existing research literature on dysmobility syndrome.

Method: All articles reporting dysmobility syndrome were identified in a systematic review of Medline (Proquest), CINAHL, PubMed, PsycInfo, EMBASE, and Scopus databases. Key characteristics of identified studies were extracted and summarized.

Results: The systematic review identified five papers (three cross-sectional, one case control, and one longitudinal study). No intervention studies were identified. Prevalence of dysmobility syndrome varied between studies (22\%-34\% in three of the studies). Dysmobility syndrome was shown to be associated with reduced function, increased falls and fractures, and a longitudinal study showed its significant association with mortality.

Conclusion: Early research on dysmobility syndrome indicates that it may be a useful classification approach to identify older people at risk of adverse health outcomes and to target for early interventions. Future research needs to standardize the optimal mix of measures and cut points, and investigate whether balance performance may be a more useful factor than history of falls for dysmobility syndrome.

Keywords: mobility, elderly, functional decline

\section{Introduction}

Increasing age is associated with a decline in function of all systems involved in physical and cognitive functions. However, it is important to differentiate decline in these systems that is purely associated with normal aging, from that caused by health conditions (both diagnosed and non-diagnosed or subclinical) or through environmental and behavioral constraints (eg, sedentary behavior). While normal aging is associated with similar system declines but of a much milder magnitude, these declines do not generally impact upon daily activities and independence until advanced age. In contrast, health conditions, environmental and behavioral factors can have substantial impact on independence, function, and quality of life, even at relatively young stages of older age (eg, in the 60s).

For many years, geriatricians and gerontologists have been exploring a number of classifications in an attempt to accurately identify older people most at risk of serious declines in health, independence, and function. Accurate identification provides the potential to target appropriate and effective interventions to this cohort, aiming to slow down or reverse this decline. In particular, early recognition of decline or risk of decline provides the greatest opportunity for prevention or reversal through intervention. Over 25 years ago, the term sarcopenia was introduced following a meeting of researchers and clinicians in the USA seeking to identify and group key components of functional decline. ${ }^{1}$ There are a number of consensus definitions of sarcopenia, and although there is variability in the criteria used to define sarcopenia, each includes measures of muscle 
mass and muscle function. ${ }^{2-6}$ The focus on sarcopenia over recent years has resulted in substantial growth in descriptive, predictive, and interventional studies.

A limitation of the sarcopenia focus is that irrespective of the criteria and cut points used, an essential component of the diagnosis is the presence of low muscle mass. However, some older people with normal range muscle mass are still at high risk or do develop the adverse health outcomes discussed above, and it has been recognized that muscle mass needs to be considered among a broader range of equally relevant areas of assessment. ${ }^{7,8} \mathrm{~A}$ similar case has been put forward regarding the diagnosis of osteoporosis in isolation. ${ }^{9,10}$ Definitions of sarcopenia continue to be modified over time with additional domains of assessment. ${ }^{8}$ Several researchers have identified that although there has been substantial research published in the sarcopenia area, clinical uptake of the application of the definitions and utilization to drive clinical interventions has been relatively poor., ${ }^{49}$

During the same time period, to address the concern that the focus on only muscle strength and muscle mass was too narrow in both identifying people at risk of decline and to inform intervention development and implementation, other researchers developed the term "frailty". ${ }^{11,12}$ A number of definitions and criteria for frailty have been published. ${ }^{11-15}$ These have generally included a measure of muscle strength, but also additional factors such as loss of weight, fatigue, and low levels of physical activity.

More recently, these terms have come under criticism as they do not consider the potential interaction between conditions and may be a limiting factor in recognizing and implementing comprehensive interventions that may be effective across these disorders. ${ }^{9}$ Hence, a new term dysmobility syndrome - has been developed by Binkley et a ${ }^{9}$ to incorporate a more diverse range of factors that may be a better predictor of adverse health outcomes for older people. The six factors recommended were as follow: osteoporosis, falls in the preceding year, obesity/high fat mass, low lean mass, slow gait speed, and low grip strength. The classification of dysmobility syndrome was proposed if three or more of these factors were present. The Binkley classification did not require any essential or pre-requisite elements, so any combination of three or more of these factors is considered indicative of the presence of dysmobility syndrome. Binkley et $\mathrm{al}^{9}$ have proposed draft cut point criteria for each of these factors (Table 1).

Although Binkley et al's study ${ }^{9}$ provides a recommended framework for the factors to include in the classification of dysmobility syndrome and cut points for each
Table I Proposed factors and cut point scores for classification of dysmobility syndrome (requires three or more of the factors to be present)

\begin{tabular}{ll}
\hline Factor & Recommended cut point for impairment \\
\hline Osteoporosis & $\begin{array}{l}\text { T-score of } \leq-2.5 \text { at lumbar spine, femoral } \\
\text { neck, or total proximal femur }\end{array}$ \\
$\begin{array}{l}\text { Falls in the preceding year } \\
\text { Low lean mass }\end{array}$ & $\begin{array}{l}\text { Self-report of one or more falls } \\
\text { Appendicular lean mass } \leq 5.45 \mathrm{~kg} / \mathrm{m}^{2} \\
\text { (females) or } \leq 7.26 \mathrm{~kg} / \mathrm{m}^{2}(\mathrm{males})\end{array}$ \\
& $<1.0 \mathrm{~m} / \mathrm{s}$ (comfortable speed) \\
Slow gait speed & Hand-held dynamometer: $<30 \mathrm{~kg}$ (male); \\
Low grip strength & $<20 \mathrm{~kg}$ (female) \\
& $\begin{array}{l}\text { Total body \% fat: }>30 \text { for males: }>40 \text { for } \\
\text { Obesity/high fat mass }\end{array}$ \\
\hline
\end{tabular}

Note: Data from Binkley et al. ${ }^{9}$

of the six factors, the authors also conclude that "the factors chosen and cut points applied here are almost certainly not ideal".

The purpose of this review is to conduct a systematic review of the current published research on dysmobility syndrome.

\section{Method}

A systematic review of the Medline (Proquest), CINAHL, PubMed, PsycInfo, EMBASE, and Scopus databases was conducted, for papers published from January 1975 to August 2016. Criteria for papers to be included in the review were as follow: 1) target population was community-dwelling older people and 2) research investigating prevalence of dysmobility syndrome, factors associated with dysmobility syndrome, adverse outcomes associated with dysmobility syndrome, or evaluating an intervention for people with dysmobility syndrome. Given that this is a relatively newly described syndrome, all paper types except descriptive and review papers were included, (eg, case study, cross-sectional, longitudinal, and intervention papers were included). Only papers published in peer reviewed journals and written in English were included. Reference lists of identified papers were also searched for additional papers. The search terms used for this systematic review were dysmobility or dysmob*, and the relevant year range was also entered. The search, screen, and data extraction were undertaken by KF and replicated by EB. Any disagreement regarding inclusion or exclusion of papers was resolved by review by a third author $(\mathrm{KH})$.

Identified papers were grouped according to their type into 1) case control, cross-sectional, or longitudinal studies and 2) interventions to address dysmobility syndrome. Key characteristics of papers were extracted and summarized in tables. 


\section{Results}

Figure 1 reports the results of the systematic review search. Following the exclusion of duplicates and papers not meeting the other inclusion criteria, five papers were retained for full review. These included three cross-sectional studies, ${ }^{9,16,17}$ one case-control study, ${ }^{18}$ and one paper analyzing longitudinal data reporting information relating to dysmobility syndrome. ${ }^{19}$ There were no identified intervention studies of any nature investigating approaches to slow down or reverse progression of dysmobility syndrome. Table 2 outlines the characteristics and findings of the included studies.

\section{Population and participants}

Overall, there were 9,561 participants included in the five non-review studies. The largest study was a cross-sectional study of 6,070 females from Korea, ${ }^{18}$ while Looker included

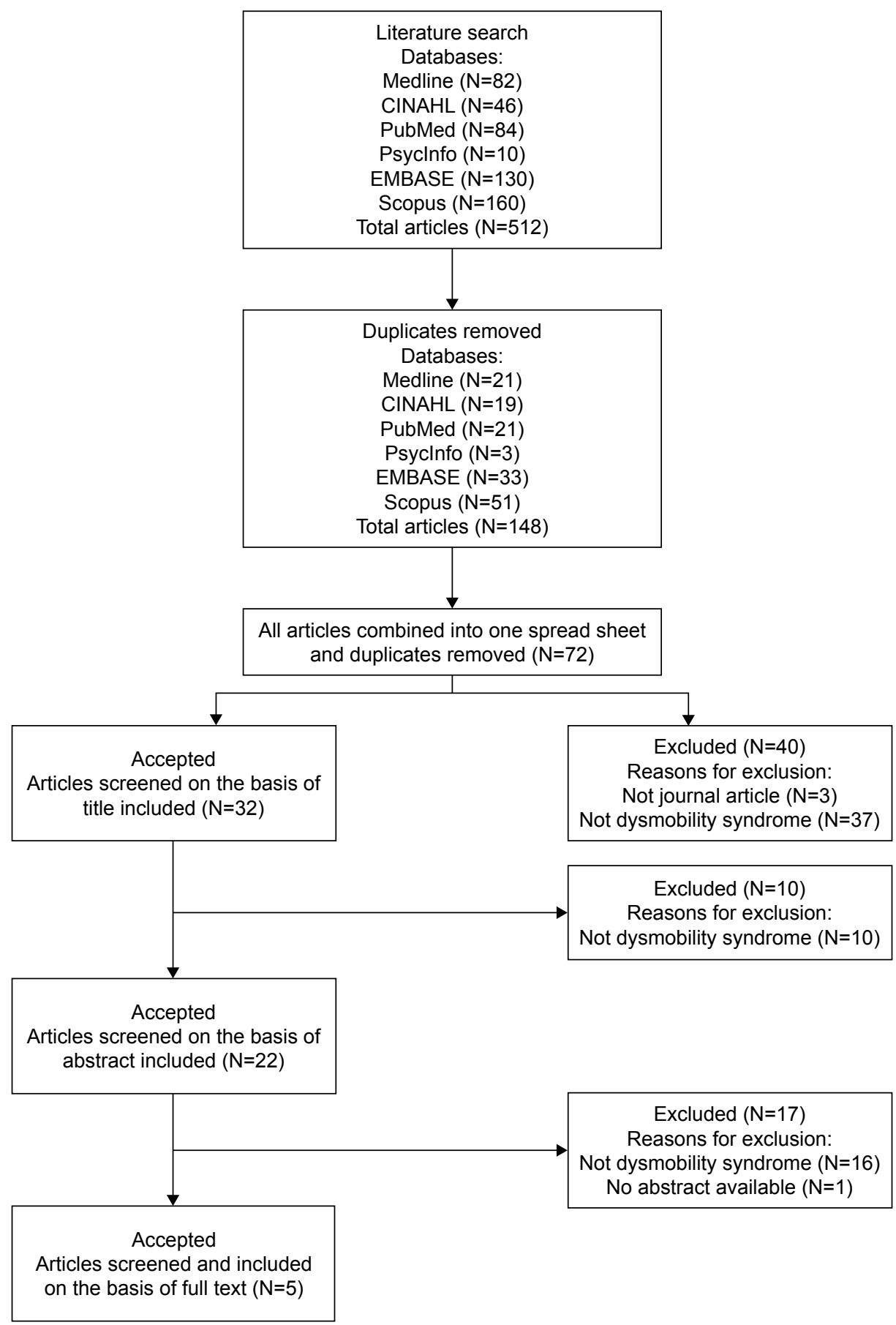

Figure I Flow chart of search results. 


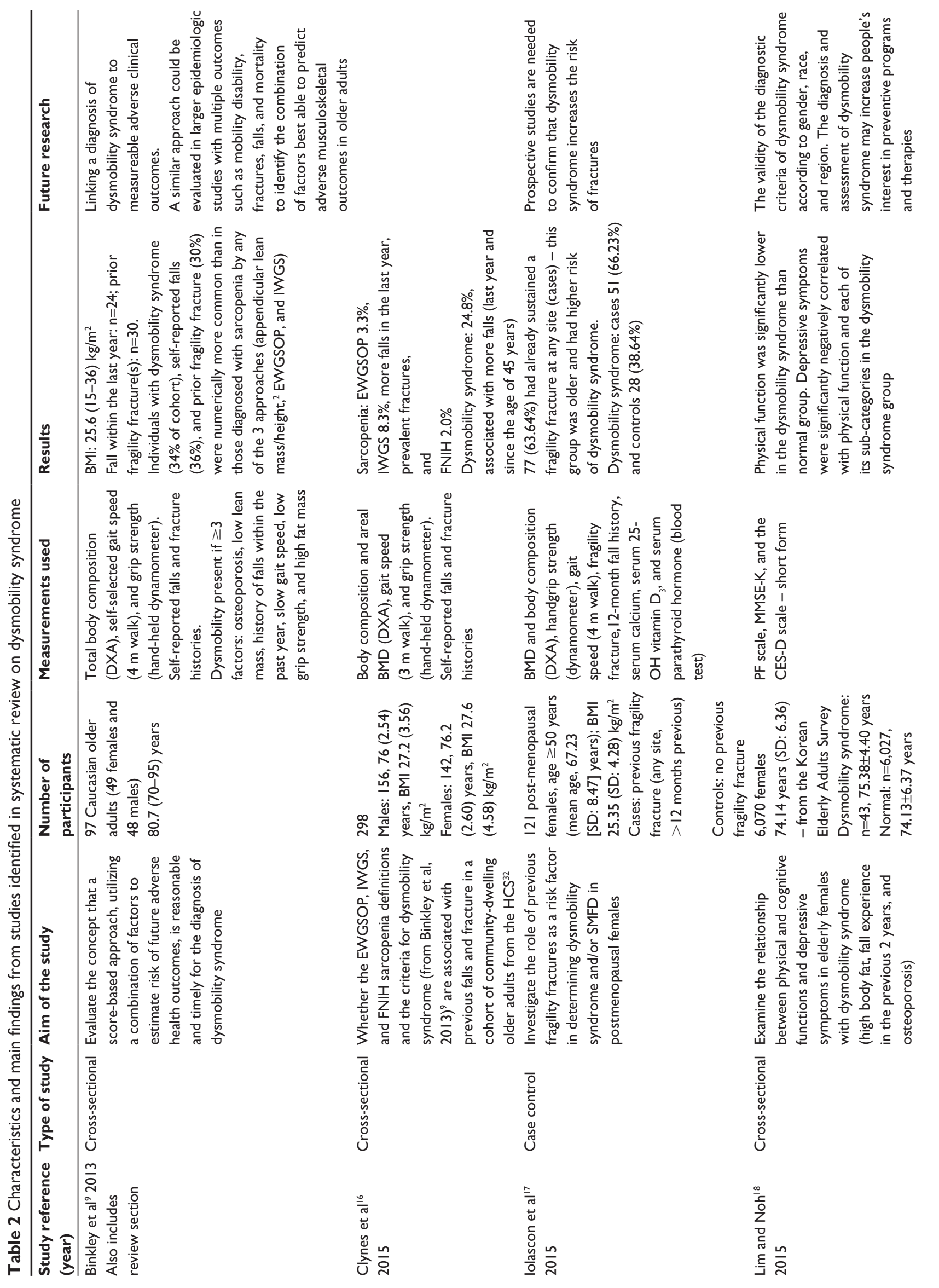



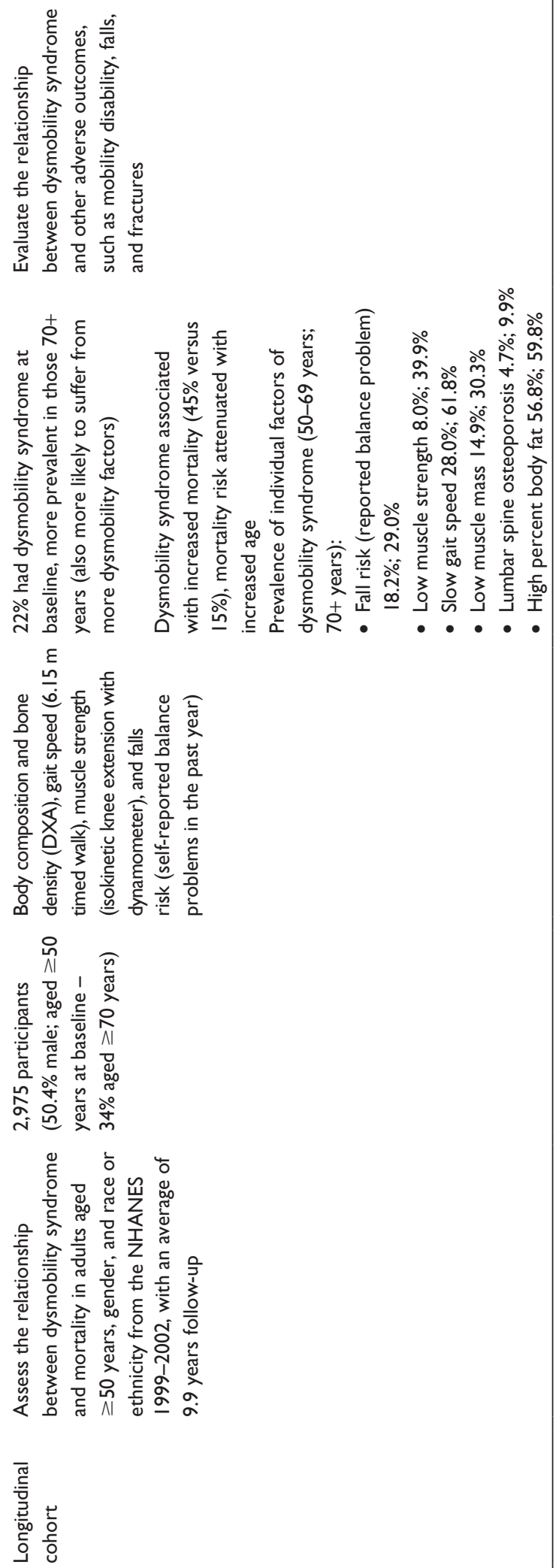

$\frac{7}{8}$
$\frac{1}{0}$
$\frac{0}{0}$
2,875 participants in his prospective cohort study based in the USA. The participants from the Looker's study were followed-up for 9-12 years and were from the National Health and Nutrition Examination Survey (NHANES) - a large representative survey of people aged $\geq 50$ years. ${ }^{19}$ Other studies had $<300$ participants and equal numbers of males and females, with the exception of the case-control study in Italy, which included females only. ${ }^{17}$ The mean age of the samples in the four studies reporting this ranged from 67.2 to 80.7 years (the other study by Looker included a sample aged $>50$ years, with $34 \%$ aged $>70$ years). ${ }^{19}$

\section{Dysmobility measures}

Most of the studies utilized variations of the measures and cut points in their dysmobility syndrome assessments to those originally reported by Binkley et al. ${ }^{9}$ In part, this may be due to several of these studies being based on secondary analyses of existing data sets, which may have utilized varying measures or cut points for a specific assessment item. Three of the papers used the same six cut points as Binkley et al, ${ }^{9}$ but Looker ${ }^{19}$ used different cut points for low muscle strength, osteoporosis, and falling risk; Ioloascon et $\mathrm{al}^{17}$ used different cut points or definitions for low lean mass, slow gait speed, low grip strength, and obesity; and Clynes et $a 1^{16}$ used a different definition for obesity. One paper ${ }^{18}$ appeared to utilize a moderately different classification system, classifying dysmobility syndrome if all three criteria of high fat mass, falls in past 2 years, and osteoporosis were present (instead of any three of the six measures reported by Binkley et al being present).

\section{Findings of the studies}

The prevalence of dysmobility syndrome differed according to measurements used in defining dysmobility syndrome and the population. It was reported to be $34 \%,{ }^{9} 24.8 \%,{ }^{16}$ and $22 \%{ }^{19}$ across three of the studies. Lim and $\mathrm{Noh}^{18}$ reported $0.7 \%$ of their sample had dysmobility syndrome, however, as noted before, their differing criteria for classification limit comparison to the other studies. An increased prevalence of dysmobility syndrome has been reported with increasing age, being present in $17.9 \%$ of those aged $70-74$ years; $24.5 \%$ of those aged $74-78$ years; and $32.1 \%$ of those aged $78-82$ years. ${ }^{9}$

In the small number of studies reporting data on dysmobility syndrome, only one has reported prevalence of each of the individual factors comprising the overall dysmobility syndrome score. ${ }^{19}$ In this sample for those aged 50-69 years, slow gait speed was present in $28.0 \%$ of the sample, low 
muscle mass in $14.9 \%$, lumbar spine osteoporosis in $4.7 \%$, and high percent body fat in $56.8 \%$. Higher prevalence of each of the component factors was evident for those aged $\geq 70$ years in this sample. Several studies also reported average scores on some or all of the individual component scores for those with and without dysmobility syndrome. The only component of dysmobility syndrome reported separately for its prevalence in the investigated sample across multiple studies was for "recent falls", which was reported as being present in between $20.7 \%$ and $25.5 \%$ of these samples. ${ }^{9,16,17}$ Looker ${ }^{19}$ reported the distribution of the number of individual component conditions making up the dysmobility syndrome score, with those aged 50-69 years having a median of one component condition, and those aged $\geq 70$ years having a median of two component conditions, and a greater proportion of the sample with three to six component conditions meeting the specified dysmobility syndrome criteria. Importantly, the study by Binkley et $\mathrm{al}^{9}$ compared the ability of two measures of sarcopenia and a measure of lean muscle mass with the use of the proposed dysmobility syndrome domains and criteria and showed that the dysmobility syndrome measure captured the majority of those identified at risk by the other measures, while also identifying a substantial different at risk population from the same sample. Similarly, the dysmobility syndrome classification appeared to identify a greater at risk population than several measures of skeletal muscle function deficit, including sarcopenic skeletal muscle function deficit. ${ }^{17}$

Several studies investigated the association between the presence of dysmobility syndrome and other indicators of adverse health outcomes. The cross-sectional study by Lim and Noh identified significantly lower physical function for the small subsample with dysmobility syndrome. ${ }^{18}$ Several studies reported falls or fracture rates for participants meeting the criteria for dysmobility syndrome: Iolascon et $\mathrm{al}^{17}$ reported increased risk of fragility fractures (odds ratio [OR], 2.46; 95\% confidence interval [CI], 1.04-5.81); Clynes et al ${ }^{16}$ reported an association with falls in the last year (OR, 5.53; CI, 3.03-10.1) and falls since age 45 (OR, 2.54; CI, 1.34-4.81), but no association with fractures since age 45 .

Investigating the outcome of mortality, Looker ${ }^{19}$ followed participants from a baseline assessment of dysmobility syndrome in 1999-2001 until December 2011. Dysmobility syndrome was associated with significantly greater mortality ( $45 \%$ of those with dysmobility syndrome at baseline died, compared to $15 \%$ of those without dysmobility syndrome). A differential magnitude of risk was identified with age, with attenuation of the risk in older age groups (those aged $>70$ years).

\section{Discussion}

Dysmobility syndrome is a recently described multicomponent classification that aims to improve use in clinical practice and research of approaches to identify early those older people most at risk of future adverse outcomes and improve early implementation of effective interventions. ${ }^{9}$ This review has highlighted the small number of papers reporting any aspect of dysmobility syndrome. However, the limited research available to date provides preliminary support for this broader classification system than others that have been utilized previously, including those of sarcopenia and frailty.

The concept of dysmobility syndrome has developed from a range of other literature that has considered the need to focus more broadly on a cluster of factors that may adversely impact on health and wellbeing outcomes for older people, rather than multiple narrow factors being looked at in isolation. For example, some of the sarcopenia literature has highlighted the need for this more holistic approach. ${ }^{8,16,17,20}$ Morley et al also described "sarcopenia with limited mobility" further recognizing the importance of consideration of additional mobility domains to the sarcopenia classification. ${ }^{7}$

For widespread utilization, classifications such as this need to be 1 ) accurate; 2 ) be practical and able to be readily utilized in clinical settings; and 3) amenable to successful intervention. With respect to accuracy, there appears to be a need to more clearly define several of the contributory factors of dysmobility syndrome. The six factors defined in the Binkley et al's study initially reporting dysmobility syndrome were described in the context of:

cutpoint values are arbitrary, potentially contentious and may very well require refinement and alteration if the dysmobility syndrome concept moves forward. ${ }^{9}$

A number of the papers cited in this review have used existing data sets, which appear to have utilized in some cases measures of a factor or cut points that differ from those described by Binkley et al. ${ }^{9}$ For example, Looker utilized "risk of falling" defined as:

having problems with balance based on a single questionnaire item that asked respondents if they had dizziness, difficulties with balance or difficulties with falling in the past 12 months $^{19}$

in contrast to the factor classification by Binkley and colleagues being "history of falls within the past year"9. Such 
differences are likely to affect the research outcomes being reported. There is a need for clear operationalization and standardization of the component factors of dysmobility syndrome.

The history of falls factor does add an important consideration to those associated with muscle and bone. However, history of falls can be problematic. Up to $20 \%$ of older people forget falls, particularly minor falls, when asked about falls in the preceding 12 months. ${ }^{21}$ Furthermore, many older people do not report falls to their health practitioners, for various reasons. ${ }^{22}$ In addition, by the time a fall occurs, often a moderate level of falls risk has developed. There may be greater value in identifying older people with increasing falls risk before a fall actually occurs. One or more measures of balance performance might be useful to consider to complement or be included instead of the existing measure of 12-month history of falls. However, further research is required to determine whether a measure of balance performance might yield better discrimination than the history of falls items. If this were to occur, there would be a need to determine the most useful single test of standing balance performance, of the many tests available. ${ }^{23}$

There is also considerable discussion about the most useful cut point for at least one of the six factors - gait speed, with most recommendations for cut points of $1 \mathrm{~m} / \mathrm{s}$ or $0.8 \mathrm{~m} / \mathrm{s}^{2,7,8}$ In recent years, an Asian consensus report on sarcopenia has recommended the cut point of $0.8 \mathrm{~m} / \mathrm{s},{ }^{6}$ in contrast to the $1.0 \mathrm{~m} / \mathrm{s}$ recommended by the International working group ${ }^{2}$ and the European working group. ${ }^{24}$ The Asian modified cut point on this and other domains evaluated for the diagnosis of sarcopenia were developed based on Asian data, reflecting different performance outcomes (in part likely to be related to different anthropometric measures).

Of note, there were no intervention studies identified in the systematic review. A recent systematic review highlighted that a number of exercise interventions were effective in improving outcomes for older people with sarcopenia, although nutrition interventions were equivocal. ${ }^{4}$ The exercise interventions for sarcopenia primarily focus on muscle strength training. However, there is strong research evidence particularly for exercise-related interventions to improve a range of other adverse outcomes for at risk older people, including falls and fractures,${ }^{25}$ balance performance, ${ }^{26}$ and function. ${ }^{27}$ However, specificity of training and ensuring safety, particularly when incorporating balance-related exercises, mean that care needs to be applied in prescribing appropriate exercises for the desired outcomes. Most likely of benefit across a range of domains would be multimodal exercises that include some balance, strength training, and cardiovascular fitness exercises. These type of exercise programs have been shown to be safe and feasible and effective in improving physical performance in older people with mild levels of functional impairment, ${ }^{28}$ through to samples with more advanced health problems ${ }^{29}$ and those with cognitive impairment. $^{30,31}$

A limitation of the emerging research in the area of dysmobility syndrome is that although there has been general consistency in the six factors included in the classification, there have been somewhat varied approaches to ascertaining the presence or absence of the factor, which is likely to impact on the classification accuracy and ability to make direct comparisons between studies. The research to date provides preliminary support for the wider application of dysmobility syndrome in research, and for consideration for clinical practice, however, there will need to be more stringent operational definitions utilized for each of the factors.

In summary, the small amount of research investigating dysmobility syndrome since it was originally described by Binkley et $\mathrm{al}^{9}$ in 2013 suggests that it may be a useful classification system warranting further investigation. There may be value in considering the use of a single standing balance test item in place of, or as well as the falls history indicator, given some limitations noted with this factor. There is a need for further research to achieve standardization of definitions and cut points to determine the value of the dysmobility classification system in accurate and early classification of risk of future adverse health outcomes and effectiveness of interventions to improve longer-term outcomes for those classified with dysmobility syndrome.

\section{Disclosure}

The authors report no conflicts of interest in this work.

\section{References}

1. Rosenberg IH. Sarcopenia: origins and clinical relevance. J Nutr. 1997; 127(Suppl 5):990s-991s.

2. Fielding RA, Vellas B, Evans WJ, et al. Sarcopenia: an undiagnosed condition in older adults. Current consensus definition: prevalence, etiology, and consequences. International working group on sarcopenia. J Am Med Dir Assoc. 2011;12(4):249-256.

3. Muscaritoli M, Anker SD, Argiles J, et al. Consensus definition of sarcopenia, cachexia and pre-cachexia: joint document elaborated by Special Interest Groups (SIG) "cachexia-anorexia in chronic wasting diseases" and "nutrition in geriatrics". Clinical Nutrition. 2010;29(2) 154-159.

4. Cruz-Jentoft AJ, Landi F, Schneider SM, et al. Prevalence of and interventions for sarcopenia in ageing adults: a systematic review. Report of the international sarcopenia initiative (EWGSOP and IWGS). Age Ageing. 2014;43(6):748-759 
5. Studenski SA, Peters KW, Alley DE, et al. The FNIH sarcopenia project: rationale, study description, conference recommendations, and final estimates. J Gerontol A Biol SciMed Sci. 2014;69(5):547-558.

6. Chen LK, Liu LK, Woo J, et al. Sarcopenia in Asia: consensus report of the Asian working group for sarcopenia. J Am Med Dir Assoc. 2014; 15(2):95-101

7. Morley JE, Abbatecola AM, Argiles JM, et al. Sarcopenia with limited mobility: an international consensus. J Am Med Dir Assoc. 2011; 12(6):403-409.

8. Edwards $\mathrm{MH}$, Buehring B. Novel approaches to the diagnosis of sarcopenia. J Clin Densitom. 2015;18(4):472-477.

9. Binkley N, Krueger D, Buehring B. What's in a name revisited: should osteoporosis and sarcopenia be considered components of "dysmobility syndrome?". Osteoporos Int. 2013;24(12):2955-2959.

10. Lewiecki EM, Bilezikian JP, Binkley N, et al. Update on osteoporosis from the 2014 Santa Fe Bone symposium. Endocr Res. 2015;40(2): $106-119$.

11. Rockwood K, Song X, MacKnight C, et al. A global clinical measure of fitness and frailty in elderly people. CMAJ. 2005;173(5):489-495.

12. Fried LP, Ferrucci L, Darer J, Williamson JD, Anderson G. Untangling the concepts of disability, frailty, and comorbidity: implications for improved targeting and care. J Gerontol A Biol Sci Med Sci. 2004;59(3): 255-263.

13. Cesari M, Nobili A, Vitale G. Frailty and sarcopenia: from theory to clinical implementation and public health relevance. Eur J Intern Med. 2016;35:1-9.

14. Dent E, Kowal P, Hoogendijk EO. Frailty measurement in research and clinical practice: a review. Eur J Intern Med. 2016;31:3-10.

15. Theou O, Brothers TD, Pena FG, Mitnitski A, Rockwood K. Identifying common characteristics of frailty across seven scales. J Am Geriatr Soc. 2014;62(5):901-906.

16. Clynes MA, Edwards MH, Buehring B, Dennison EM, Binkley N, Cooper C. Definitions of sarcopenia: associations with previous falls and fracture in a population sample. Calcif Tissue Int. 2015;97(5): 445-452.

17. Iolascon G, Moretti A, Giamattei MT, Migliaccio S, Gimigliano F. Prevalent fragility fractures as risk factor for skeletal muscle function deficit and dysmobility syndrome in post-menopausal women. Aging Clin Exp Res. 2015;27(Suppl 1):S11-S16.

18. Lim E, Noh J. Physical function, cognitive function, and depressive symptoms in elderly women with dysmobility syndrome. Int J BioSci Bio Technol. 2015;7(4):229-238.

19. Looker AC. Dysmobility syndrome and mortality risk in US men and women age 50 years and older. Osteoporos Int. 2015;26(1):93-102.
20. Chung JH, Hwang HJ, Shin HY, Han CH. Association between sarcopenic obesity and bone mineral density in middle-aged and elderly Korean. Ann Nutr Metab. 2016;68(2):77-84.

21. Hill K, Schwarz J, Flicker L, Carroll S. Falls among healthy, communitydwelling, older women: a prospective study of frequency, circumstances, consequences and prediction accuracy. Aust $N Z$ J Public Health. 1999;23(1):41-48.

22. Lee DC, Day L, Hill K, Clemson L, McDermott F, Haines TP. What factors influence older adults to discuss falls with their health-care providers? Health Expect. 2015;18(5):1593-1609.

23. Huxham FE, Goldie PA, Patla AE. Theoretical considerations in balance assessment. Aust J Physiother. 2001;47(2):89-100.

24. Cruz-Jentoft AJ, Baeyens JP, Bauer JM, et al. Sarcopenia: European consensus on definition and diagnosis: report of the European working group on sarcopenia in older people. Age Ageing. 2010;39(4):412-423.

25. Gillespie LD, Robertson MC, Gillespie WJ, et al. Interventions for preventing falls in older people living in the community. Cochrane Database Syst Rev. 2012;12(9):CD007146.

26. Howe T, Rochester L, Neil F, Skelton D, Ballinger C. Exercise for improving balance in older people. Cochrane Database Syst Rev. 2011;(11): CD004963.

27. Liu C, Latham NK. Progressive resistance strength training for improving physical function in older adults. Cochrane Database Syst Rev. 2009; (3):CD002759.

28. Yang XJ, Hill K, Moore K, et al. Effectiveness of a targeted exercise intervention in reversing older people's mild balance dysfunction: a randomized controlled trial. Phys Ther. 2012;92(1):24-37.

29. Sherrington C, Tiedemann A, Fairhall N, Close JC, Lord SR. Exercise to prevent falls in older adults: an updated meta-analysis and best practice recommendations. N SW Public Health Bull. 2011;22(3-4):78-83.

30. Burton E, Cavalheri V, Adams R, et al. Effectiveness of exercise programs to reduce falls in older people with dementia living in the community: a systematic review and meta-analysis. Clin Interv Aging. 2015;10: $421-434$

31. Suttanon P, Hill KD, Said CM, et al. Feasibility, safety and preliminary evidence of the effectiveness of a home-based exercise programme for older people with Alzheimer's disease: a pilot randomized controlled trial. Clin Rehabil. 2013;27(5):427-438.

32. Syddall HE, Sayer AA, Simmonds SJ, et al. Birth weight, infant weight gain, and cause-specific mortality: the Hertfordshire Cohort Study. Am Journal Epidemiol. 2005;161(11):1074-1080.
Clinical Interventions in Aging

\section{Publish your work in this journal}

Clinical Interventions in Aging is an international, peer-reviewed journal focusing on evidence-based reports on the value or lack thereof of treatments intended to prevent or delay the onset of maladaptive correlates of aging in human beings. This journal is indexed on PubMed Central, MedLine,

\section{Dovepress}

CAS, Scopus and the Elsevier Bibliographic databases. The manuscript management system is completely online and includes a very quick and fair peer-review system, which is all easy to use. Visit http://www.dovepress. com/testimonials.php to read real quotes from published authors. 